Felicia Logozzo \& Paolo Poccetti (eds.)

Ancient Greek Linguistics: new approaches, insights, perspectives

De Gruyter Studium 
Seriestitle 
Felicia Logozzo \& Paolo Poccetti (eds.)

Ancient Greek

Linguistics: new

approaches, insights,

perspectives

DE GRUYTER 


\section{Classification}

35-02, 65-02, 65C30, 65C05, 65N35, 65N75, 65N80

ISBN 978-3-11-055175-4

\section{(c) BY-NC-ND}

This work is licensed under the Creative Commons AttributionNonCommercial-NoDerivs 3.0 License. For details go to http://creativecommons.org/licenses/by-nc-nd/3.0/.

\section{Library of Congress Cataloging-in-Publication Data}

A CIP catalog record for this book has been applied for at the Library of Congress.

\section{Bibliographic information published by the Deutsche Nationalbibliothek}

The Deutsche Nationalbibliothek lists this publication in the Deutsche Nationalbibliografie; detailed bibliographic data are available on the Internet at http://dnb.dnb.de.

(C) 2017 Copyright-Text, published by Walter de Gruyter GmbH, Berlin/Boston The book is published with open access at www.degruyter.com.

Cover image: Cover-Firma

Typesetting: le-tex publishing services $\mathrm{GmbH}$, Leipzig

Printing and binding: Printing binding

(2) Printed on acid-free paper

Printed in Germany

www.degruyter.com 
Dedication can be placed here 



\section{Contents}

Massimo Vai

Struttura informativa della frase in greco omerico: periferia alta, periferia bassa;

collocazione delle relative nella periferia sinistra -1 



\title{
Struttura informativa della frase in greco omerico: periferia alta, periferia bassa; collocazione delle relative nella periferia sinistra
}

\begin{abstract}
L’analisi cartografica della periferia sinistra della frase può essere utilmente adottata per rendere conto della struttura informativa della frase nel greco antico. Oltre alla periferia sinistra "alta”, già individuata a partire dagli studi di Hale (1987) (per le lingue indoeuropee) e da Rizzi (1997) e Benincà (2001) (per l'italiano e altre lingue romanze), a partire da Belletti (2004) viene individuata anche una periferia bassa, anch'essa articolata in proiezioni di topic e focus. Il presente studio si propone di integrare, entro un unico quadro sintattico teorico, ciò che sembra più chiaro, allo stato attuale, nell'analisi della struttura della frase nel greco antico, e in particolar modo nel greco omerico. Infatti, la ricerca intorno alla struttura della frase del greco antico, soprattutto a partire da Dik (1995; 2007), Matić (2003), Dal Lago (2010) e Bertrand (2010), ha conosciuto momenti di maggior definizione rispetto ai precedenti tentativi, che spesso non erano andati al di là di un'analisi in termini di “ordine libero" dei costituenti. Una volta stabiliti alcuni punti fermi nella struttura, è anche possibile verificare l'interazione fra la struttura informativa e la collocazione di alcune proposizioni: qui si prendono in considerazione alcuni tipi di correlative.
\end{abstract}

\section{Quadro teorico: periferia sinistra ${ }^{1}$ della frase e periferia “bassa” (Low Periphery).}

Con periferia sinistra della frase intendiamo indicare un'organizzazione interna in termini di proiezioni ordinate in cui si pensa sia articolata la precedente proiezione di CP (Split CP), pioneristicamente a partire da Hale (1987) per le lingue indoeuropee antiche, e successivamente da Rizzi (1997), Benincà (2001) in termini più generali.

1 Nonostante questa denominazione abbia avuto illustri critici, che l'hanno attribuita a una visione della lingua determinata dal verso della scrittura degli autori in cui questa denominazione è stata adottata, resta il fatto che, se si considera il tempo di esecuzione di una frase su un asse ordinato $t_{1}<t_{2}$ ecc., ecco che il concetto diventa indipendente dalla forma di scrittura adottata: ciò che è a sinistra precede nel tempo ciò che è a destra. 
Lo Split-CP è pensato come insieme ordinato di proiezioni, a loro volta ordinate in campi (field), cioè in insiemi di proiezioni che condividono proprietà, ovvero specifiche caratteristiche semantiche e sintattiche. I campi, individuati da $\{\ldots\}$, contengono proiezioni singole, indicate da [...]:

$$
\text { Frame }[\mathrm{HT}][\text { Sc Sett }]_{\text {Topic }}[\mathrm{LD}][\mathrm{LI}]_{\text {Focus }}[\text { Foc contr] }[\text { Foc inf }] / \text { [Interr wh-].... }
$$

Inoltre, Benincà - Poletto (2004) hanno individuato all'interno del campo Topic due proiezioni specifiche, indicate come [LD] e [LI]. All'interno di questo quadro, i processi di focalizzazione e tematizzazione sono visti come prodotti dal movimento di costituenti negli specificatori delle proiezioni dedicate dello Split-CP: l'interpretazione di focalizzazione e tematizzazione è pensata in termini simili ai processi di accordo.

Più recentemente, a partire da Belletti (2004) e, oltre ad altri, Cognola (2008) e Poletto $(2014,55)$, è stata avanzata anche l'ipotesi di una periferia bassa di VP (low periphery):

[vP [Topic1 [Topic2 [Topic3... [Operator... VP]...] ${ }^{2}$

per giustificare ordini marcati in italiano antico, ad es:

il quale da che ebbe tutto Egitto vinto,... (Bono Giamboni, Orosio)

mentre per il resto mostra comportamenti da lingua VO.

\section{Struttura informativa della frase nel greco antico}

L’approccio pragmatico fa del greco antico una lingua FWO (free word order), nella quale l'ordine dei costituenti è determinato da ragioni comunicative e non è legato a fattori sintattici.

Nel modello adottato anche da Dik (1995) il Focus (narrow focus) deve precedere il Verbo; Focus e Verbo, inoltre, devono essere adiacenti. Lo schema che viene proposto è il seguente:

(1) $\mathrm{P} 1-\mathrm{P} \emptyset-\mathrm{V}-\mathrm{X}$

Topic Focus Verb pragmatically unmarked

Matić $(2003,578)$ ritiene che il modello in 1) non riesca a rendere conto di alcuni dati:

1. vi sono elementi postverbali che non possono essere considerati unmarked (da lui considerati postverbal foci);

2. più di due soli elementi preverbali (complexity of the left periphery);

2 Schema tratto da Poletto $(2014,55)$. 
3. elementi compresi fra focus e verbo (focus intruders).

Dik $(2007,38)$ ha modificato il suo schema originario in questi termini:

(2) Setting-Topic-Focus-Verb-Remainder

In 2) la periferia sinistra della frase è stata incrementata con l'ulteriore posizione di Setting ${ }^{3}$. Comune allo schema di Matić è la collocazione immediatamente preverbale del (narrow) focus, quindi ad es.:

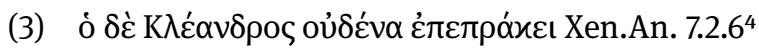

'Quanto a Cleandro, non (ne) aveva venduto nessuno'.

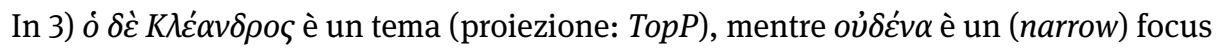
(proiezione: FocP).

Tuttavia Matić ritiene che anche il segmento postverbale vada analizzato, contrariamente a Dik, la quale ritiene che questa parte della frase non sia suscettibile di ulteriore analisi, facendo essa parte di ciò che Dik definisce come remainder, cioè la parte presupposta dell'enunciato. Matić osserva che il quadro teorico di Dik non può rendere conto dei molti casi in cui la porzione postverbale dell'enunciato dovrebbe essere analizzata come broad focus ("focus esteso"), comprendente il predicato e alcuni argomenti, come in:

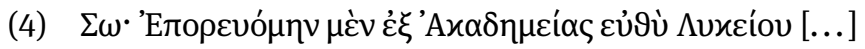

'Andavo dall'Accademia verso il Liceo'

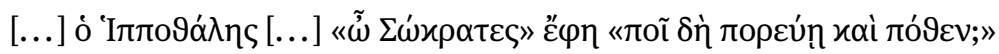

'Ippotale disse «O Socrate, dove vai e da dove vieni?'

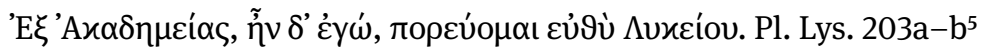

'«Dall'Accademia» dissi io «sto andando verso il Liceo»'

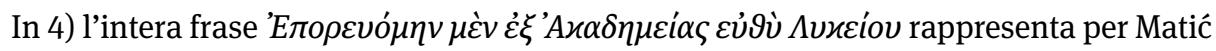

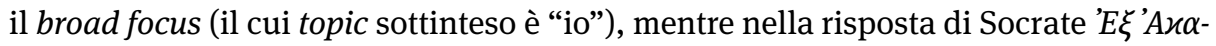
$\delta \eta \mu \varepsilon i ́ \alpha \varsigma$ costituisce il narrow focus collocato, come di norma, in posizione preverbale.

In altri casi, in cui la parte presupposta è già ricavabile dal contesto, Matić (2003: 588) analizza ulteriormente il broad focus, ad es.:

3 La questione è stata ripresa anche da Lühr (2009) con le seguenti osservazioni:

i) enclitici e parole con tono basso sono preferibilmente in seconda posizione (Legge di Wackernagel);

ii) la negazione è all'inizio della frase o precede immediatamente il verbo;

iii) quando una parola è enfatizzata può essere separata dal suo costituente (iperbato);

iv) il verbo di una frase principale tende a collocarsi in mezzo alla frase, risultando negli ordini:

S-Pred-O, O-Pred-S (se l'oggetto è enfatizzato), Avv - Pred - S;

v) se parte di una frase complessa è enfatizzata, di solito è collocata all'inizio.

4 Matić $(2003,575)$.

5 Matić $(2003,583)$. 
(5) [Contesto:

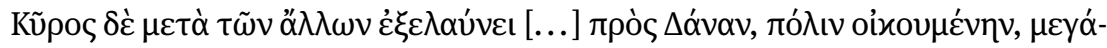

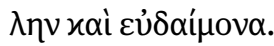

'Ciro, con il resto dell'esercito marcia [...] verso Dana, città popolosa, grande e prospera']

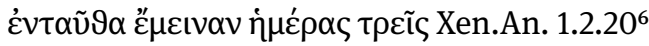

'Qui restarono per tre giorni'

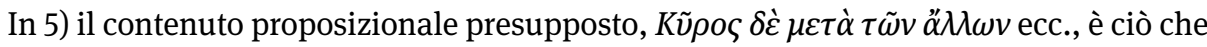

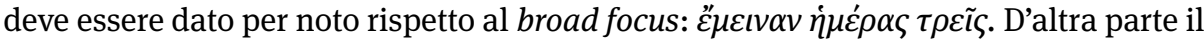
broad focus è ulteriormente analizzabile in [qui rimasero per un tempo $\mathrm{x}]_{\text {presupposto }} \mathrm{e}[\mathrm{x}$ $=$ tre giorni $]_{\text {asserito }}$. In questo modo, la gran parte degli elementi postverbali può essere analizzata, mostrando l'esistenza di un focus "basso" postverbale.

Inoltre, Matić (2003, 587; 591-593) osserva che talvolta ciò che fa parte del contenuto presupposto è collocato immediatamente dopo il verbo, ad es.:

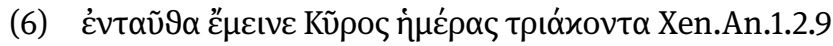

'Qui Ciro rimase trenta giorni'

In 6) Kũ $\rho$ ऽ rappresenta l'elemento che riprende, come topic postverbale, la narrazione interrotta.

Riassumendo, Matić (2003) mostra che nel greco antico, in posizione postverbale, esiste uno spazio sintattico in cui vengono collocati gli elementi tematizzati e focalizzati di ciò che da Belletti (2004) viene chiamata low periphery.

Dal Lago (2010) $)^{7}$ ha sviluppato un criterio di analisi sintattica della periferia sinistra della frase in greco antico che utilizza la collocazione delle particelle $\mu \varepsilon^{\prime} v$ e $\delta \varepsilon ́$ : questi elementi vengono utilizzati come punto di riferimento per effettuare una dettagliata analisi della periferia sinistra in Senofonte, soprattutto per il field di Topic. Come marcatori di List-IinterpretationP $(L I P)^{8}$, le particelle $\mu \varepsilon \dot{v}$ e $\delta \varepsilon$ occorrono in posizione Wackernagel, cioè dopo la prima parola della frase o dopo il primo costituente, oppure più in là nella frase, nel caso in cui la periferia sinistra contenga elementi tematizzati, segnatamente vocativi, avverbiali e Hanging Topic. Le due particelle $\mu \varepsilon \dot{v}$ e $\delta \dot{\varepsilon}$, posposte ai costituenti interessati, costituiscono nella maggioranza dei casi i marcatori della posizione di LIP, che ospita costituenti tematizzati contrastivi. A livello sintattico, inoltre, la presenza nell'enunciato di costituenti in LIP possono essere di vari tipi: il costituente in LIP, di volta in volta, può infatti essere l'oggetto, il verbo, un DP o un PP.

6 Matić $(2003,585)$.

7 In Vai (2003, 79-80) avevo già connesso le due particelle alla List InterpretationP di Benincà-Poletto allora in corso di stampa, poi 2004. Certamente Dal Lago (2010) ne ha tratto più numerose conseguenze. 8 Cfr. Benincà - Poletto (2004, 67-70). 
In greco antico possiamo trovare esempi che ci indicano elementi in caso accusativo sicuramente tematizzati, che però non sono ripresi da alcun pronome, come accade invece, a parità di struttura informativa, in italiano:

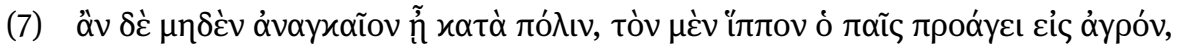

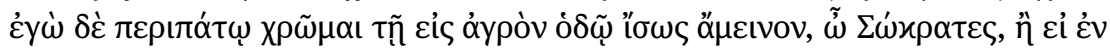

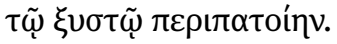

'Nel caso in cui non ci sia nulla di pressante in città, il cavallo lo porta lo schiavo in campagna, io invece vado a piedi in campagna...' Xen.Oec. $11.15^{9}$

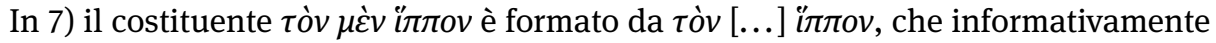
è un elemento tematizzato, come è indicato dalla presenza di $\mu \varepsilon ́ v$, e precede l'altro

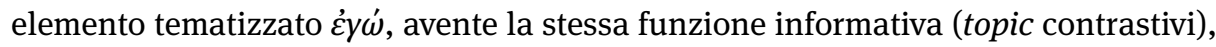
indicata dalla presenza di $\delta \dot{\varepsilon}$.

\section{Struttura informativa della frase nel greco omerico $^{10}$}

\subsection{Periferia sinistra “alta” (High Left Periphery)}

\subsubsection{Elementi dei field di Frame e Topic}

In greco omerico esiste una periferia sinistra “alta” della frase molto articolata. Nel seguente esempio compare un tema sospeso seguito da elementi tematizzati della LIP caratterizzati da $\mu \varepsilon^{v} v$ e $\delta \varepsilon$ come in greco classico:

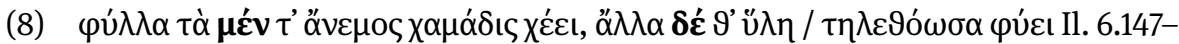
148

'Le foglie, alcune (ne) getta a terra il vento, altre la selva fiorente (ne) produce'

In 8) le particelle $\mu \varepsilon^{\prime} v$ e $\delta \varepsilon ́$ vengono collocate secondo la Legge di Wackernagel ( $L W$ ). Wackernagel (1892, 371; 377) classifica queste particelle fra i sonstige Enklitika o QuasiEnklitika o fra le postpositive Partikeln, dal momento che, benché accentate, sembrano seguire la stessa regola di collocazione dei clitici veri e propri. Nel caso osservato, $\mu \varepsilon ́ v$, nonostante non occorra linearmente dopo la prima parola, né dopo il primo costituente

9 Citato in Dal Lago (2010, 23).

10 Bertrand (2010) rappresenta un saggio molto dettagliato sull'ordine dei costituenti in greco antico e, in particolare, nel greco omerico, basato principalmente sul quadro teorico di Dik - Matić: vi farò in parte riferimento nella mia analisi. 
della frase in cui compare, non rappresenta un'eccezione alla LW: come già osservato in Hale (1987), la collocazione dei clitici W in seconda posizione va intesa non tenendo conto di eventuali costituenti iniziali tematizzati. Così ad es. anche in vedico (qui il clitico asya compare linearmente in terza posizione):

$\begin{array}{llll}\text { (9) indrah } & \text { kím } & \text { asya sakhyé } & \text { cakāra RV 6.27.1b } \\ \text { Îndra-NOM } & \text { cosa-ACC } & \text { di-lui amicizia-LOC } & k r \text {-3SGPF }\end{array}$

'Indra, cosa ha fatto nella sua amicizia?'

Anche in latino (il clitico me compare come seconda parola del costituente nulla cupiditas, dopo il costituente tematizzato de triumpho autem):

(10) de triumpho autem nulla me cupiditas umquam tenuit

'Riguardo al trionfo non mi ha mai preso nessuna voglia'. Cic. Att. 7.2.6

In greco classico, la collocazione dopo il primo costituente o dopo la prima parola si traduce nel fatto che, nei DP con articolo, le particelle si inseriscono o all'interno del sintagma tra articolo e nome, oppure dopo il sintagma costituito da articolo + nome, ad es.:

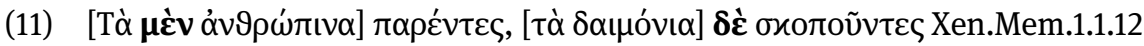
'Le cose umane trascurando, le divine, invece, osservando'.

La collocazione dei clitici della LW dopo prima parola o dopo primo costituente è nota anche nelle lingue moderne che collocano i clitici analogamente, ad es ${ }^{11}$. in serbocroato:

(12) [Anina sestra] im nudi čokoladu [Anina im sestra] nudi čokoladu 'di-Anna la sorella offre loro cioccolato'.

In 8) (Il. 6.147-148), prima degli elementi tematizzati introdotti da $\mu \varepsilon^{\prime} v$ e $\delta \varepsilon ́$ compare $\varphi v ́ \lambda \lambda \alpha$ come ulteriore costituente tematizzato: con tutta probabilità si tratta di un Hanging Topic, che è previsto occorrere a sinistra degli elementi in LIP (entro il field di Frame).

In altri casi, a sinistra di LIP si trovano elementi di Scene Setting, sempre entro il field di Frame:

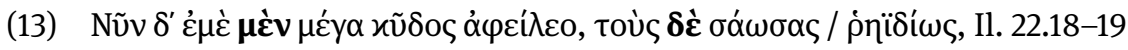
'Così ora, a me, (mi) hai tolto una grande gloria, quelli invece (li) hai salvati / senza fatica'.

In 13) gli elementi di LIP sono $\varepsilon \dot{\varepsilon} \dot{\varepsilon} \mu \grave{\varepsilon} v . . . \tau o u ̀ \varsigma ~ \delta \dot{\varepsilon}$, a sinistra dei quali compare $N \tilde{v} \nu \delta^{\prime}$ in ScSetting.

Anche il contesto Top - wh individua come topic i costituenti che precedono un elemento interrogativo:

11 Tratto da Progovac $(1996,415)$. 


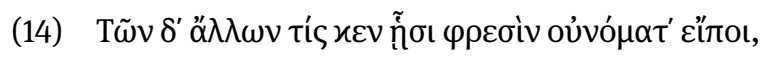

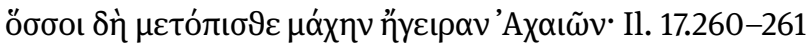

'Ma degli altri, chi potrebbe nella sua mente dire i nomi, quanti dei Danai dietro destarono battaglia?'.

Rientrano fra i casi di topic anche le occorrenze di verbo in prima posizione $\left(\mathrm{V}_{1}\right)$ dovute a topic nullo (Ø) - cfr. Matić $(2003,582)$, ad es.:

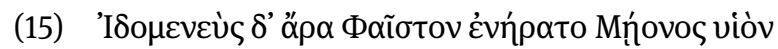

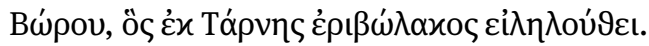

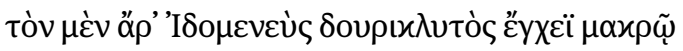

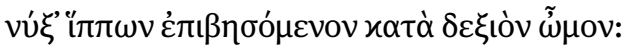

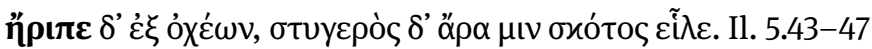

'Idomeneo trafisse Festo, figlio del meonio Boro, il quale era giunto da Tarne fertile zolla; costui Idomeneo buona lancia con l'asta lunga (lo) trafisse, mentre saliva sul carro, nella spalla destra; Ø cadde dal carro, lo prese la tenebra odiosa'.

In 15) si è verificato un mutamento di topic, da Idomeneo a Boro, segnalato da $\tau \grave{\partial} v \mu \varepsilon \dot{v} v$ al v. 45; lo stesso Boro continua a essere associato al topic nullo del v. 47.

\subsubsection{New Topic e riattivazione di topic}

New topic è un elemento che risponde al concetto di aboutness propria dei topic, ma che non era ancora stato introdotto nel discorso (cfr. Bertrand 2010: 159-160), ad es.:

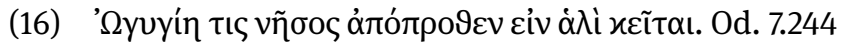

'C’è un'isola, Ogigia, lontana nel mare'.

Come in 16), Bertrand osserva che in questi casi il greco omerico utilizza spesso una costruzione presentativa. Dal punto di vista informazionale è simile a questo è il caso di un topic che viene reintrodotto in questa funzione associato a un elemento che era già presente nel discorso, ma molto lontano, ad es.:

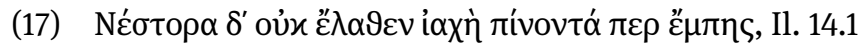

'Non sfuggì a Nestore il grido, benché stesse bevendo'

Nestore era già comparso nell'opera, ma in 17) viene citato all'inizio di un canto. Questo caso è frequente anche in vedico:

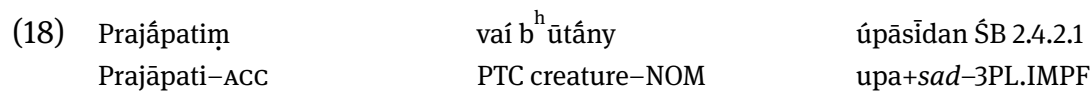

'(Una volta) a Prajāpati si avvicinarono le creature'.

In 18) Prajāpati, personaggio già noto, è reintrodotto nel racconto come topic di una nuova storia. 


\subsubsection{Elementi del field di Focus}

Matić (2003, 582 ss.) e Bertrand (2010, 96 ss.) distinguono due tipi di focus: broad focus/domaine focal e narrow focus/focus restreint. Il primo costituito dal verbo e dai suoi argomenti, il secondo limitato a un solo argomento. Rientrano in quest'ultimo caso i costituenti focalizzati a sinistra del verbo flesso, corrispondenti alla posizione di narrow focus di Matić $(2003,588)$ :

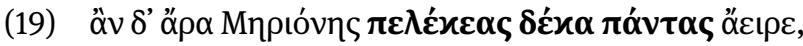

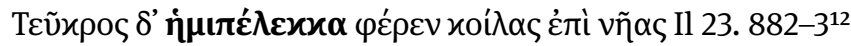

'Merione dunque le dieci doppie scuri si prese, Teucro portò le scuri alle navi'.

In 19) Merione e Teucro si trovano in posizione di topic (probabilmente LIP), mentre in

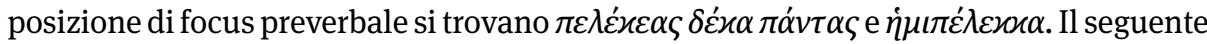
caso è interessante per la collocazione di "Nessuno":

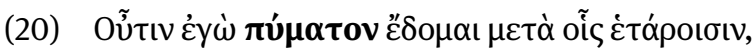

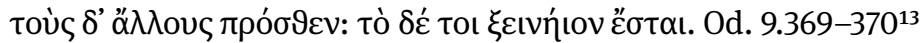

'Nessuno io (lo) mangerò per ultimo, dopo i compagni

Gli altri invece prima: questo sarà il tuo dono ospitale'.

La collocazione di $\pi v ́ \mu \alpha \tau o v$ è in posizione di focus preverbale, a destra di $O \tilde{v} \tau \iota v$, che va

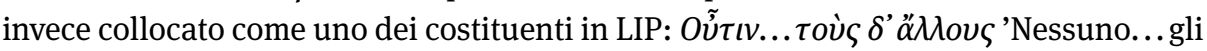
altri invece...' In 20) “nessuno” è un topic proprio a causa dell'inganno di Odisseo, mentre normalmente il quantificatore si troverebbe in focus, ad es.:

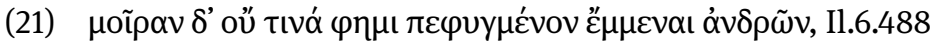
'ma alla Moira, NESSUNO - (ti) dico - è sfuggito, degli uomini'.

In 21) abbiamo $\mu о \tilde{\imath} \rho \alpha \nu \delta^{\prime}$ in topic, mentre ov̉ $\tau \iota v \alpha$ si trova regolarmente in (narrow) focus.

Altri ess. introdotti da elementi focalizzatori:

(22) тoì yà

'Perciò io ANCHE QUESTO ti dirò molto sinceramente'.

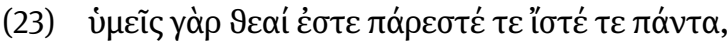

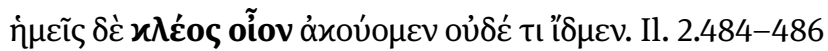

'voi, dee, voi siete sempre presenti, sapete tutto, noi invece SOLO LA FAMA ascoltiamo e non abbiamo visto niente'.

12 Citato in Bertrand (2010, 120).

13 Citato in Bertrand $(2010,121)$. 


\subsection{Periferia bassa (Low Periphery)}

\subsubsection{Topic postverbale}

Come si è detto, anche in posizione immediatamente postverbale, nella periferia bassa, si trovano costituenti identificabili come tematizzati. In particolare, Matić (2003: 587; 591-593) osserva che talvolta ciò che fa parte del contenuto presupposto è collocato immediatamente dopo il verbo, mentre Bertrand (2010, 197-198) parla, a questo proposito parla di topique ratifié:

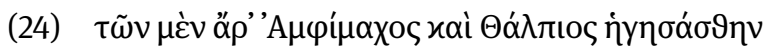

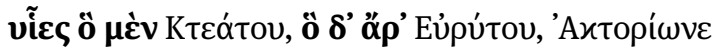

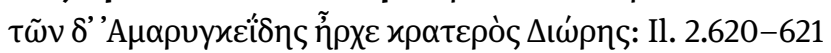

'Erano dunque a capo degli uni Anfimaco e Talpio, uno figlio di Crèato, l'altro di Eurito, degli altri era a capo il forte Diore Amarincide'.

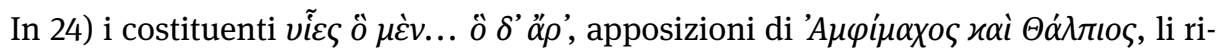
prendono come topic collocati dopo il verbo $\dot{\eta} y \eta \alpha \dot{\alpha} \sigma \vartheta \eta v$ : anche in questo caso il ruolo informativo è indicato dalla successione di $\mu \varepsilon^{\prime} v$ e $\delta \varepsilon$.

Un altro caso può essere il seguente (da Bertrand 2010, 198):

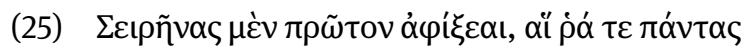

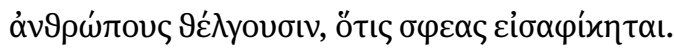

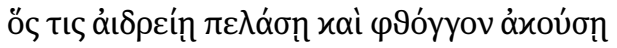

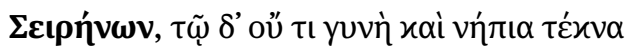

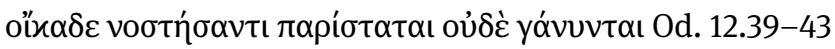

'Alle Sirene prima giungerai, che tutti gli uomini stregano, chi le avvicini. Chi ignaro approdi e ascolti la voce delle Sirene, mai più la moglie e i figli infanti, tornato a casa, festosi lo attorniano'.

\subsubsection{Focus postverbale}

Anche per la periferia bassa è disponibile una posizione di focus, che spesso, insieme al verbo che lo precede, forma il broad focus. Dal punto di vista sintattico si tratta spesso di soggetti nella sequenza $V-S$ che segue a un altro argomento del verbo in posizione di topic preverbale (Matić $(2003,585)$ :

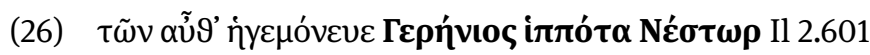
'di questi era a capo il gerenio cavaliere Nestore'.

Si noti che, quando la successione è $\mathrm{S}$ - V, la frase può contenere un narrow focus:

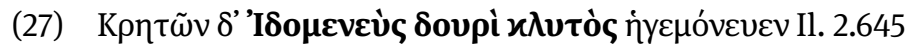

'Sui Cretesi comandava Idomeneo buono con l'asta'. 
Tuttavia altre volte il soggetto postverbale costituisce una ripresa, quindi si tratta più probabilmente di un topic (cfr. Bertrand 2010, 207-209), ad es.:

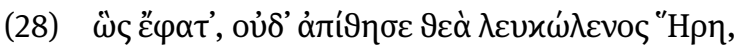

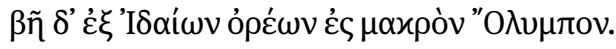

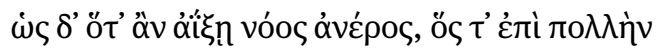

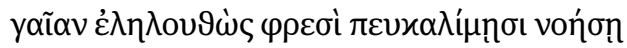

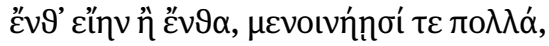

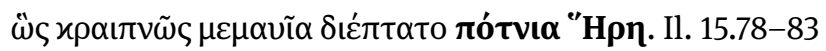

'Così parlò [scil. Zzúç], non mancò di ascoltare la dea Era braccio bianco: e si diresse dalle alte cime dell'Ida verso il grande Olimpo. Come quando si lancia la mente di un uomo, che, avendo percorso molta terra, pensa nei suoi pensieri acuti: "fossi là, oppure là", e pensa molte cose, così velocemente volò bramosa, Era santissima'.

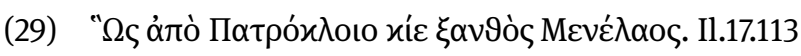

‘Così si allontanò da Patroclo, il biondo Menelao’.

In altri casi il focus postverbale consiste dell'oggetto o di altri argomenti del verbo, oppure anche di avverbi e aggettivi predicativi (Bertrand 2010, 97-98):

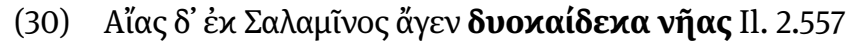

'Aiace da Salamina guidava dodici navi'.

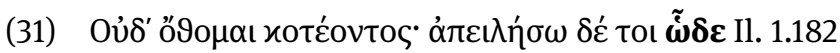

'Non ti temo adirato, ma ti dico questo: [...]'

Altrove il focus è introdotto o caratterizzato da elementi focalizzatori (ad es.: "anche”, "da solo"):

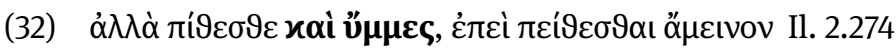

'obbedite anche voi, perché è meglio obbedire'

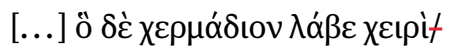

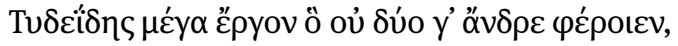

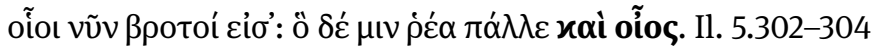

'L'altro prese un masso con le mani, il Tidide - grande azione! - che oggi non porterebbero in due, per come sono gli uomini di adesso: lui invece lo brandiva senza fatica anche DA SOLO'.

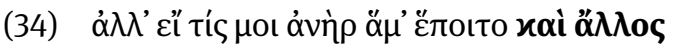

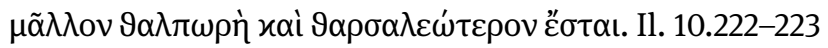

'Ma se venisse con me ANCHE UN ALTRO, ci sarà più speranza e maggiore coraggio'. 


\subsection{Excursus. ő = sá figé?}

Bertrand $(2010,164)$ osserva che la ripresa di un topic può realizzarsi anche attraverso il pronome ö. Particolarmente interessante è il caso (l'unico, informa Bertrand) di ö che si accorda con la 1a.sg. del verbo:

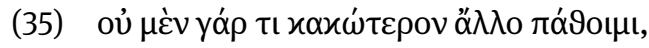

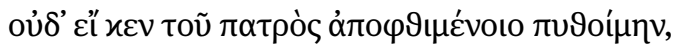

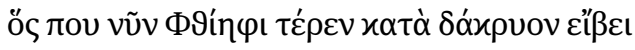

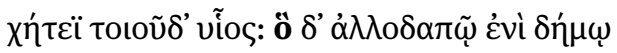

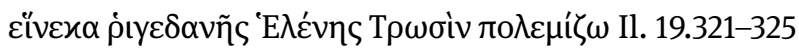

'Mai soffrirò disgrazia più grave, nemmeno se di mio padre morto sapessi - che forse a Ftia una tenera lacrima versa per la mancanza di un tale figlio: (io) che, in paese straniero per la funesta Elena combatto coi Troiani'.

Il fatto è già stato osservato da Dunkel 1990 a proposito del sá figé. La denominazione di sá figé14 si riferisce a un insieme di passi in cui la forma del pronome vedico sá, sấ, tád compare soprattutto al nom. sg. masch., in posizione iniziale di frase, apparentemente senza referente, tuttavia evidenziabile in presenza di accordo del verbo alla 1a o 2a persona. Questa particolarità dell'accordo, già notata da Delbrück $(1888,211)$, aveva indotto Watkins a pensare a un'antico connettivo sá divenuto molto comune in nessi come sá yádi, sá yátra, sá céd. Vi sono ess. di tale uso in RV e in Śatapathabrāhmana, ad es.:

(36) sa yády anuláb eran prasromtamātrám vā_ñjalimātrám vā ŚB 4.5.10.7 ${ }^{15}$ 'se prendono la misura di una o due mani'.

(37) nấ_nyấ yuvát non_altra voi-DU-ABL

prámatir sollecitudine-NOM asti máhyam è $a-m e$

$\begin{array}{llll}\text { sá vāṃ } & \mathrm{d}^{\mathrm{h}} \text { íyam } & \text { vājayántīm } & \text { atakșam//RV 1.109.1c } \\ \text { sá voi-DU-ACC } & \text { pensiero-ACC } & \text { gratificante-ACC } & \text { creai }\end{array}$

'Non c'è altra cura per me che voi due, così (?) ho creato per voi due un pensiero gratificante'.

Talvolta viene usata anche la forma flessa del pronome sá-/tá-:

14 Per una rassegna critica del problema si rinvia a Berenguer Sánchez (2000, 104 ss.).

15 Citato in Berenguer Sánchez $(2000,105)$. 
(38) RV 5.24

\begin{tabular}{|c|c|c|c|}
\hline ágne & tváṃ & no & ántama \\
\hline Agni-voc & $\mathrm{tu}-\mathrm{NOM}$ & di-noi & intimo-NOM \\
\hline utá trātầ & \multirow{2}{*}{\multicolumn{2}{|c|}{$\begin{array}{l}\text { śivó } \\
\text { benevolo-NOM }\end{array}$}} & b avā varūt yàh / \\
\hline e salvatore-NOM & & & sii protettore-NOM \\
\hline vásur & \multicolumn{2}{|l|}{ agnír } & vásuśravā \\
\hline buono-NOM & \multicolumn{2}{|l|}{ Agni-NOM } & beni-famoso-NOM \\
\hline ác ${ }^{\mathrm{h}} \bar{a}$ nakṣi & dyumáttamạ̣ & rayím & dāḥ / \\
\hline qui giungi-IMP & luminosa-ACC & ricchezza-ACC & dà \\
\hline sá no & $\operatorname{bod}^{\mathrm{h}} \mathrm{i}$ & śrud ${ }^{\mathrm{h}} \hat{\mathrm{i}}$ & hávam \\
\hline sá di-noi & sii-attento & ascolta & voce-ACc \\
\hline urusyấ no & $\mathrm{ag}^{\mathrm{h}}$ āyatáh & & samasmāt / \\
\hline proteggi noi & malevolo-ABL & & chiunque-ABL \\
\hline tám tvā & śocisțt ${ }^{\mathrm{h}} \mathrm{a}$ & & dìdivah \\
\hline tá-ACc te & splendente-voc & & lucente-voc \\
\hline sumnâya & nūnám īmahe & & sák ${ }^{\mathrm{h}}$ ib yah / \\
\hline benevolenza-DAT & ora veniamo & & compagni-DAT \\
\hline
\end{tabular}

'O Agni, sii tu nostro amico e salvatore benevolo protettore. Agni è buono e è famoso per i beni: giungi qui e dacci la tua luminosa ricchezza. (Tu essendo tale?) abbi riguardo per noi, ascolta la nostra voce, proteggici da ogni malevolo. (Come tale?) a te ora veniamo, o splendente lucente, per la benevolenza verso (noi) tuoi compagni’.

Non è chiaro se si debba pensare a un uso di sá connettivo come a un esito di grammaticalizzazione a partire da un pronome flesso, oppure se si tratti di un arcaismo, precedente lo sviluppo delle forme flesse - in ciò accordandosi con il connettivo itt. $\check{s} u^{16}<{ }^{\star} s o$ - che sarebbero sorte dalla fusione del connettivo con l'antico pronome enclitico, ancora attestato da itt. $-a \check{S}^{17}<{ }^{\star}-o s$.

16 Cfr. Hoffner - Melchert (2008, 392-393).

17 Cfr. Hoffner - Melchert $(2008,135)$. 


\section{Relative in greco omerico e periferia sinistra della frase}

\subsection{Problemi nella ricostruzione del pronome relativo i.e.}

Dal punto di vista morfologico, fino alla scoperta dell'ittita era opinione prevalente che il pronome relativo originario del proto-i.e. fosse $*(H)$ io- e che solo successivamente in alcune lingue il pronome interrogativo e indefinito ${ }^{\star} k^{\hat{u}} i-/ k^{u} 0-$ avrebbe sostituito l'altro anche in funzione di relativo. Ovviamente la scoperta dell'ittita e, più recentemente, del celtiberico hanno mutato la prospettiva della ricostruzione: la protolingua utilizzava già questi elementi come relativi, e, se sì, solo uno o entrambi?

\section{A IPOTESI: *(H)।O-}

Il maggior argomento nel considerare ${ }^{\star}(H)$ io- come l'antico relativo è il fatto che i riflessi diretti di questo elemento compaiono come pronomi o come complementatori connessi a pronomi di originario valore relativo (cfr. Probert 2015, 24-27): a.sl.eccl. i-že (rel), ide 'dove', jegda 'se', jako ‘che'; got. jabai ‘se, quando'; lit. jéi/jéigù ‘se’, jóg 'che'. Dal momento che molti complementatori si basano sul tema del relativo (cfr. lat. quod 'il fatto che', 'poiché'), questo sembrerebbe un ulteriore argomento per supporre l'esistenza di un relativo * $(H)$ io - in i.e. Tuttavia, nonostante gli indizi di germ. e baltosl., il maggior argomento a favore di questo pronome è l'isoglossa congiuntiva ario-grecoceltica (che costituisce geolinguisticamente l'area maggiore): infatti la scoperta delle iscrizioni del celtiberico di Botorrita ha posto una seria riserva all'ipotesi nel frattempo avanzata che * $(H)$ io - fosse un'innovazione orientale rispetto ad un supposto più antico ${ }^{*} K^{u} i-/ k^{u} O_{-}$, ad es:

(39) iomui lisTaś TiTaś sisonTi śomui $[\ldots]^{18}$

REL-DATSG ACCPL ACCPL V3PL DIM-DAT SG

'A chi... a colui...'

2A IPOTESI: * $\mathbf{K}^{\mathrm{U}} \mathbf{I}-/ \mathrm{K}^{\mathrm{U}} \mathrm{O}-$

Il maggior argomento che ${ }^{\star} k^{u} i-/ k^{u} o$ - fosse già pronome relativo in i.e. è basato sulla sua presenza in anatolico e in latino-sabellico (Probert 2015: 27-32). Altrove lo stesso tema compare come indefinito e interrogativo, funzione, quest'ultima, da cui può tipologicamente derivare quella di relativo. Come i pronomi originati da * $(H){ }_{\alpha} i_{-}$, anche quelli derivati da ${ }^{\star} k^{u} i-/ k^{u} O-$ compaiono storicamente attestati in strutture correlative.

18 Citato da Ziegler (1993, 252). 


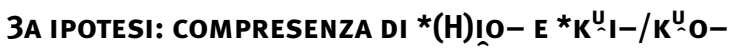

Altri pensano che i due temi potessero coesistere nella protolingua, ma con funzioni differenziate: ${ }^{*} k^{u} i-/ k^{u} o$ - in relative di tipo correlativo e ${ }^{*}$ Hio- in relative appositive (Probert 2015, 32 ss.). La presenza di abbondante documentazione in greco e in vedico di relative posposte all'antecedente e di valore non restrittivo sembra avvalorarne l'arcaicità, mentre è tipologicamente inusuale che le non restrittive precedano la principale. Se si accetta questa ipotesi, allora in greco e in indo-iranico ${ }^{*} \mathrm{Hio}-$ avrebbe sostituito ${ }^{*} k^{u} i-/ k^{u} o-$ nelle funzioni di correlativo. Che questo possa accadere sembra confermato dal fatto che, in greco omerico, anche il dimostrativo-anaforico ò, ì, tó $\grave{\varepsilon}$ usato in relative, soprattutto posposte alla principale, ma talvolta prende anche il posto di ǒs, ň, ő in strutture correlative (v. Probert 2015, 47). La difficoltà dell'ipotesi di due tipi di relative introdotte rispettivamente dai due diversi pronomi sta nel fatto che (quasi) nessuna lingua i.e. avrebbe mantenuto l'originaria distribuzione di ${ }^{*} \mathrm{Hio}-\mathrm{e}$ ${ }^{*} k^{u} i-/ k^{u} o-$ (Hettrich $1988,770-771$ menziona alcuni probabili casi di ${ }^{*} k^{u} i-/ k^{u} 0-$ entro il territorio di ${ }^{*}$ Hio, ad es. tess. $x$ (s). Si deve comunque notare che in greco la distinzio-

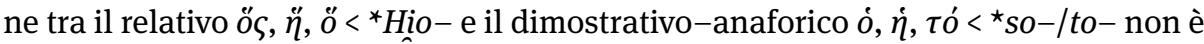
sempre esente da problemi: Probert $(2015,121-122 ; 127)$ osserva che, data la recenziorità dell'uso dell'accento, è spesso difficile distinguere tra i due pronomi, tranne che nelle forme non ambigue a prescindere dall'accento. Inoltre la distinzione fra restrittive e non restrittive, che talvolta è affidata alla sola presenza della virgola, dipende anche in questo caso dalle scelte dell'editore del testo.

\subsection{Correlative}

La frase correlativa è ampiamente rappresentata nelle fasi arcaiche di molte lingue i.e. e rappresenta con tutta probabilità un modello sintattico conservativo ascrivibile alla protolingua. In particolare, le correlative di interpretazione restrittiva, sia nelle lingue che usano ${ }^{*} \mathrm{HiO}-$ sia in quelle che usano ${ }^{*} K^{u} i-/ k^{u} \mathrm{o}$-, si caratterizzano per essere preposte alle rispettive principali e per avere testa interna. Particolarmente chiari sono alcuni casi del vedico:

\begin{tabular}{|c|c|c|c|}
\hline $\begin{array}{l}\text { (40) } \text { yámị }_{\mathbf{i}} \\
\text { quale-ACC }\end{array}$ & $\begin{array}{l}\text { yajñáạ }_{\mathbf{i}} \\
\text { sacrificio-ACC }\end{array}$ & $\begin{array}{l}\text { náyathā } \\
\text { conducete }\end{array}$ & $\begin{array}{l}\text { nara } \\
\text { Signori-VOC }\end{array}$ \\
\hline âdityā & rojúnā & & pathấ \\
\hline Āditya-VOC & retto-STRUM & & percorso-STRUM \\
\hline prá vaḥ & $\mathbf{s a ́}_{\mathbf{i}}$ & dhītáye & naśat RV 1.41.5 \\
\hline PREV voi-ACC & quello-NOM & attenzione-DAT & raggiunga-ING \\
\hline
\end{tabular}

'Il sacrificio che voi conducete, Signori Āditya, per la retta via, quello raggiunga la vostra (benevola) attenzione'. 
(41) yó

ya-NOM

mấ nah

NEG noi-GEN mártyah

mortale-NOM

sá

quel-NOM śiśsite áty

affila PREV

ripúr

ingannatore-NOM aktúbhir

notti-STRUM

î́ata RV 1.36.16

signoreggi-ING

'Il mortale che affila (le armi) nelle notti, quell'ingannatore non abbia potere su di noi'.

Anche nel greco omerico si trovano queste strutture:

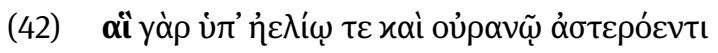

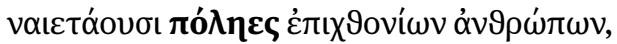

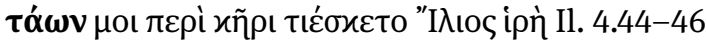

'Perché quante città di uomini terrestri che si trovano sotto il sole e il cielo stellato, fra queste Ilio sacra mi onorava di cuore'.

Tuttavia esistono, sia in vedico sia in greco, relative a testa interna posposte:

(43) sá $\mathrm{g}^{\mathrm{h}} \overline{\mathrm{a}}$

quel PTC

yám

rel-ACC

sómo

Soma-NOM viró

uomo-NOM

índro

Indra-NOM

hinóti

favorisce ná riṣyati

NEG è-danneggiato

bráhmaṇas pátị

Brahmaṇaspati-NOM

mártyam RV 1.18.4

mortale-ACC

'Quel mortale non è danneggiato, colui che Indra, Brahmaṇaspati e Soma favoriscono'.

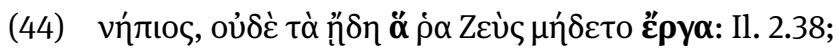

'Stolto! Non sapeva quali opere Zeus meditava'

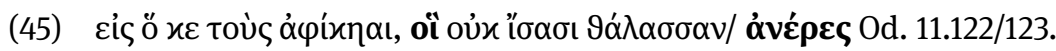

'Finché tu arrivi a uomini che non conoscono il mare'.

\subsection{Collocazione delle correlative nella periferia sinistra della frase}

Probert (2015, 311-312) osserva che la struttura delle correlative si presta particolarmente alla collocazione in posizione di topic. Questo si nota sia in vedico, sia in greco omerico: 
(46)

$\begin{array}{lll}\begin{array}{l}\text { ágne } \\ \text { Agni-VOC }\end{array} & \begin{array}{l}\text { yám } \\ \text { ya-ACC }\end{array} & \begin{array}{l}\text { yajñám } \\ \text { cerimonia-ACC }\end{array} \\ \begin{array}{l}\text { adhvarám } \\ \text { sacrificale-ACC }\end{array} & \begin{array}{l}\text { viśvátah } \\ \text { da-ovunque }\end{array} & \begin{array}{l}\text { paribhû́ ási } \\ \text { circondante-NOM sei }\end{array} \\ \text { sá } & \text { íd devéșu } & \text { gachati RV 1.1.4s } \\ \text { quello-NOM } & \text { PTC dèi-LOC } & \text { va }\end{array}$

'Agnì, quella cerimonia sacrificale che tu circondi da ogni parte, proprio quella va agli dèi'.

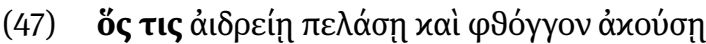

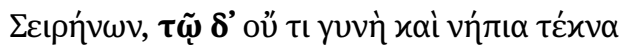

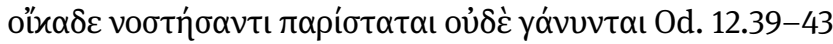

'Colui che, per non saperlo, approda e sente la voce delle Sirene, quello mai più la moglie e i figli infanti, tornato a casa, festosi lo attorniano'.

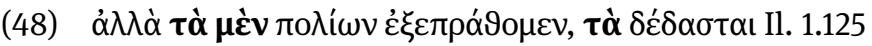

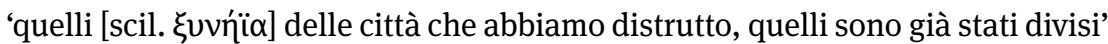

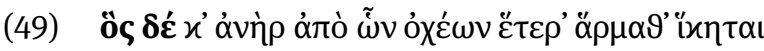

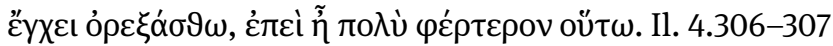

'L'uomo che possa dal proprio carro raggiungerne un altro, balzi in avanti con la lancia, perché così sarà molto meglio'.

In 46) - 49) le correlative sono collocate in posizione di topic nella periferia alta della frase. La frase correlata è introdotta in entrambe le lingue dal pronome sá/tá. In 48) si osserva che anche la correlativa è introdotta dallo stesso pronome sá/tá, mentre in 49) la principale è pro-drop.

Inoltre, in 48) la prima occorrenza di $\tau \grave{\alpha}$ potrebbe essere sostituita da $\ddot{\alpha}$, ed entrambe potrebbero aver sostituito ${ }^{*} k^{u} i-/ k^{u} 0-$ nella sua funzione originaria di correlativo.

\section{Conclusioni}

L’approccio pragmatico fa del greco antico una lingua FWO (free word order), nella quale l'ordine dei costituenti è determinato da ragioni comunicative e non è legato a fattori sintattici. Nel modello adottato anche da Dik (1995) il Focus deve precedere il Verbo; Focus e Verbo, inoltre, devono essere adiacenti. Matić (2003) ha modificato questo quadro incrementando questo schema con l'aggiunta di un'ulteriore proiezione nella periferia sinistra della frase e distinguendo fra un focus ristretto (narrow), che deve precedere il verbo flesso e un focus esteso (broad), che comprende il verbo flesso e un focus postverbale. Inoltre ha individuato un'ulteriore posizione di topic in sede 
immediatamente postverbale. Quest'analisi può utilmente essere confrontata con lavori di indirizzo generativo (Rizzi 1997, Benincà 2001) che hanno individuato anche per altre lingue una periferia sinistra "alta" articolata in field di frame, topic e focus e, a partire da Belletti (2004), un'ulteriore periferia bassa, anch'essa articolata in proiezioni di topic e di focus. Dal momento che questi ultimi studi prevedono, nelle diverse zone individuate, la collocazione di proiezioni massimali di vario tipo, è possibile pensare che anche costituenti di livello proposizionale, non solo nominale, siano adatti a occupare proiezioni di topic, focus, ecc. Si vede così che, ad es. anche le proposizioni relative possono essere pensate occupare posizioni dedicate nella periferia sinistra, in particolare qui si è considerato il caso delle correlative, la cui collocazione è in stretta associazione con la struttura informativa dell'intero periodo in cui compaiono.

\section{Bibliografia}

Belletti, A. 2004: Aspects of the Low IP Area, in The Structure of CP and IP - The Cartography of Syntactic Structures vol 2, ed. L. Rizzi, Oxford - New York, 16-51.

Benincà, P. 2001: The Position of Topic and Focus in the left periphery, in Current Studies in Italian Syntax offered to Lorenzo Renzi, eds. G. Cinque \& G. Salvi, Amsterdam, 39-64.

Benincà, P. \& Poletto, C. 2004: Topic, Focus, and V2: Defining the CP Sublayers, in The Structure of CP and IP - The Cartography of Syntactic Structures vol 2, ed. L. Rizzi, Oxford - New York, 52-75.

Berenguer Sánchez, J. A. 2000: Estudio sobre las partículas indoeuropeas con base consonántica y laringal, Madrid.

Bertrand, N. 2010: L'ordre des mots chez Homère, Doct-Thèse, Université de Paris-Sorbonne.

Cognola, F. 2008: OV/VO Syntax in Mòcheno Main Declarative Clauses, «RGG», 33, 79-93.

Dal Lago, N. 2010: Fenomeni di prolessi (pro)nominale e struttura della periferia sinistra nel greco di Senofonte, Doct-Th, Università di Padova.

Delbrück, B. 1888: Altindische Syntax, Halle.

Dik, H. 1995: Ancient Greek Word Order, Amsterdam.

Dik, H. 2007: Word Order in Greek Tragic Dialogue, Oxford.

Dunkel, G. E. 1990: J. Wackernagel und die idg. Partikeln *só, ${ }^{*} k e,{ }^{*} k e m$ und ${ }^{*} a n$, in: Sprachwissenschaft und Philologie: Jacob Wackernagel und die Indogennanistik heute, eds. by H. Eichner \& H. Rix, (Kolloquium der Indogermanischen Gesellschaft) Wiesbaden, 100-130.

Hale, M. 1987: Notes on Wackernagel's Law in the language of the Rigveda, in Studies in Memory of Warren Cowgill (1929-1985). Papers from the Fourth East Coast Indo-European Conference, Cornell University, June 6-9, 1985, ed. C. Watkins, Berlin - New York, 38-50.

Hoffner, H. A. \& Melchert, C. 2008: A Grammar of the Hittite Language. Part I: Reference Grammar, Winona Lake, Indiana.

Hettrich, H. 1988: Untersuchungen zur Hypotaxe in Vedischen, Berlin - New York.

Lühr, R. 2009: Information Structure in Ancient Greek, in The discourse potential of underspecified structures, ed. A. Steube, Berlin - New York, 487-512.

Matić, D. 2003: Topic, focus, and discourse structure, «Studies in Language», 27/3, 573-633. 
Poletto, C. 2014: Word Order in Old Italian, Oxford - New York.

Probert, P. 2015: Early Greek Relative Clauses, Oxford.

Progovac, L. 1996: Clitics in Serbian/Croatian: Comp as the Second Position, in Approaching Second. Second Position Clitics and Related Phenomena, eds. A. L. Halpern \& A. M. Zwicky, Stanford, California, 411-428.

Rizzi, L. 1997: The fine structure of the left periphery, in Elements of Grammar, ed. L. Haegeman, Dordrecht, 281-337.

Vai, M. 2003: Alcune osservazioni sull'enclisi in serbocroato, «Quaderni Patavini di Linguistica», 19, 71-113.

Wackernagel, J. 1892: Über ein Gesetz der indogermanischen Wortstellung, «Indogermanische Forschungen», 1, 333-436.

Ziegler. S. 1993: Zur Entwiklung der Relativsätze mit dem Relativpronomen *[E03A?]o- in den keltischen Sprachen, «MSS», 54, 251-270. 